\title{
Key Strategies for Building Research Capacity of University Faculty Members
}

\author{
Laura F. Huenneke ${ }^{1}$ - Diane M. Stearns ${ }^{2}$ • \\ Jesse D. Martinez ${ }^{3}$ Kelly Laurila ${ }^{4}$
}

Published online: 16 March 2017

(C) The Author(s) 2017. This article is published with open access at Springerlink.com

\begin{abstract}
Universities are under pressure to increase external research funding, and some federal agencies offer programs to expand research capacity in certain kinds of institutions. However, conflicts within faculty roles and other aspects of university operations influence the effectiveness of particular strategies for increasing research activity. We review conventional approaches to increasing research, focusing on outcomes for individual faculty members and use one federally-funded effort to build cancer-related research capacity at a public university as an example to explore the impact of various strategies on research outcomes. We close with hypotheses that should be tested in future formal studies.
\end{abstract}

Laura Huenneke received the Ph.D. from Cornell University, and she is former Vice President for Research, former Provost, and currently Professor of Environmental Sciences at Northern Arizona University. Her research interests span both environmental biology and STEM communication and career development.

Diane Stearns is the Associate Vice President for Research and Professor of Biochemistry at Northern Arizona University. She earned her Ph.D. in Chemistry from the University of California, Berkeley. Her research interests are in metal toxicology, and her administrative responsibilities include building institutional research capacity.

Jesse Martinez received his doctorate at the University of Nevada - Reno. He is a professor at the University of Arizona Cancer Center in the Department of Cell \& Molecular Medicine. His research interests include the molecular mechanisms that become disrupted during tumor formation and how this information may be applied to colon cancer prevention.

Kelly Laurila earned the M.A. in Applied Anthropology at Northern Arizona University, where she is a senior coordinator and serves as evaluator for the Partnership for Native American Cancer Prevention. She is committed to working with underserved populations and developing innovative evaluation strategies for career development, mentorship, and capacity building in higher education settings.

Laura F. Huenneke

laura.huenneke@nau.edu

1 School of Earth Sciences and Environmental Sustainability, Northern Arizona University, Flagstaff, AZ 86004-5694, USA

2 Office of the Vice President for Research, Northern Arizona University, 86011 Flagstaff, AZ, USA

3 University of Arizona Cancer Center, 1515 N. Campbell Ave, 85724 Tucson, AZ, USA

4 Department of Anthropology, Northern Arizona University, 86011 Flagstaff, AZ, USA 
Keywords University research $\cdot$ Faculty productivity $\cdot$ Mentoring

Higher education institutions are under increasing pressure to adapt to evolving competitive and financial pressures (Christensen and Eyring 2011; Selingo 2013). In particular, in recent decades many public universities in the United States have seen substantive declines in state appropriations. Changes in institutional revenues can alter institutional priorities (Fowles 2014; Jaquette and Curs 2015), and many universities are consequently exploring more entrepreneurial activities and new collaborations or partnerships (Slaughter and Rhoades 2004).

One goal embraced by many institutions is the aim of increasing research-related revenues, especially federal funding and associated indirect cost recovery (Streharsky 1991; Warshaw and Hearn 2014). Increased research activity leads both to increased revenue and to higher rankings, in turn leading to more student applications and increased tuition (Litwin 2009). Thus many universities set goals of moving up in national research activity rankings, and institutions historically more focused on instruction similarly attempt to build research funding.

Universities pursue specific strategies for building research capacity (Fisher 2009; Hearn et al. 2006; Litwin 2009). Expanding research, however, can cause tensions with traditional expectations for teaching, service, and outreach (Neumann and Terosky 2007; Romainville 1996). Increasing research focus also presents challenges in managing risk and regulatory compliance for institutions (Rosenzweig 1987). Positive outcomes of investment in building research capacity are by no means assured (Taylor and Cantwell 2015).

Public education in the United States exemplifies the way fiscal pressures can lead to restructured university budgets and activity. As an example, the three public universities in the state of Arizona (University of Arizona, Arizona State University, and Northern Arizona University) have recently experienced some of the most substantive drops in state appropriations of any public universities in the U.S. (National Center for Educational Statistics 2015). The fiscal year 2015 all-funds operating budget for these three universities estimated the state general fund contribution as only $17 \%$ of the total (Arizona Board of Regents 2015). Meanwhile, one of the state's strategic goals for the universities is the doubling of research expenditures from 2010 to 2020. This aggressive goal necessitates explicit effort to build research capacity, particularly in an institution like Northern Arizona University (NAU). While classified as a high-research doctoral institution, NAU has historically prioritized undergraduate instruction; and the university has fewer doctoral programs than its sister institutions. Efforts to build NAU's research capacity in defined areas offer opportunities to analyze the relative effectiveness of different strategies.

One federal funding program designed to build university research capacity is the Comprehensive Partnerships to Advance Cancer Health Equity (CPACHE), which is funded by the National Cancer Institute (2015) or NCI. The program aims to increase capacity for cancer-related research at institutions serving under-represented minority populations in collaboration with NCI-designated comprehensive cancer centers. NAU has received funding since 2002 from CPACHE to support The Partnership for Native American Cancer Prevention, a collaboration with the University of Arizona's Cancer Center (UACC). The Partnership for Native American Cancer Prevention (NACP or the Partnership) connects two universities operating within the same state system under a strategic vision set by a shared Board of Regents.

Creation of partnerships or collaborations is a strategy frequently followed in order to promote institutions learning from one another and pooling expertise and resources (Kornreich 
1973). However, formal relationships do not necessarily translate to successful enhancement of performance for the partners. An earlier report from a similar CPACHE collaboration (Thompson et al. 2013) detailed challenges posed by differences in institutional cultures and described strategies for surmounting these cultural differences. Communication and respect for differences in culture, such as differences in faculty workload pressures, were reported to be the most helpful strategies.

There is broad recognition that faculty members represent one of the most important elements of research capacity for an institution (Shehzad et al. 2014) and that university policies, practices, and resources greatly shape the productivity of researchers (Harkavy and Hartley 2012; Kyvik and Aksnes 2015). The analysis we offer in this article describes challenges faced and strategies employed by the Partnership in building the research capacity and careers of participating faculty at Northern Arizona University, highlighting areas in which our strategies contrast with more conventional approaches. This review is based on evaluation of program accomplishments, rather than a formal hypothesis-driven investigation of outcomes. That is, we assess a single exemplar, which was not designed as a research-based intervention. However, we outline important hypotheses for future investigation of factors influencing researcher success.

\section{Approaches to Increasing Research Activity}

In established research institutions, expansion of research is often attempted by adding faculty members to existing units and providing mentoring and infrastructure to maximize individual success. The University of Arizona Cancer Center (UACC) has pursued this conventional approach with success; however, NAU's experiences over many years in hiring individual talented researchers have been less positive, with many failing to achieve hoped-for productivity or leaving the institution. A review of the literature concerning research development reveals several strategies aimed at improving upon the standard model: cluster hiring, revision of institutional policies and infrastructure, and systemic prioritization of research for administrators as well as for individual faculty members.

The strategy of developing research in cluster areas has demonstrated advantages for emerging research universities (Birx et al. 2013). A research cluster, a multidisciplinary team of faculty members from multiple departments focused on a common theme, provides a mechanism for leveraging regional strengths, sharing limited resources, and providing opportunities for both faculty members and students. While Partnership funds have not been adequate to support a true set of cluster hires, the collaborative efforts of NAU and UACC to address issues of cancer health equity in regional populations are designed to create some of the same advantages.

Achievements at the University of Puerto Rico illustrate how developmental funding can trigger change to promote research in an undergraduate minority-serving institution (Godreau et al. 2015). Historical challenges included insufficient infrastructure for managing grant awards, high faculty teaching loads, little inclusion of research productivity in criteria for tenure and promotion, and the absence of graduate programs and therefore graduate assistants and postdoctoral researchers to enhance productivity. Solutions included securing administrative commitment to post-award staff support, hiring research active faculty with reduced teaching loads, and 
modifying tenure and promotion criteria to reward research productivity. Research students were incorporated into mentoring communities to assist with career development experiences and resources. Over the course of a decade, the institution increased the numbers of research-active faculty members, students involved in research, peerreviewed articles, and external grant proposals.

Building research capacity in an emerging research institution also requires assessment of research management practices and identification of transitional practices to promote the evolving research agenda. Based on classifications of Bosch and Taylor (2011), early phases are marked by "hand holding" management, institutional emphasis on teaching, a faculty mindset that research is intimidating, and a centralized research mission with deans' focus on teaching output and research activity rather than research quality and outcomes. Transition through a "broadening" phase to the "honing" phase is marked by increased emphasis on research relative to teaching, decentralization of the research mission into college and department priorities, increased collaboration, and a growing focus on recruitment of high quality researchers and postgraduate students. We describe how our use of institutional administrators and external advisors contributed to institutional buy-in for the shifting research agenda and how development of institutional leaders from within NAU faculty contributed to increased research capacity.

\section{Program Context and Data Sources}

Our overall question is whether NACP efforts to build cancer-related research capacity have been successful at the level of individual faculty members at NAU. We review the basic program elements that have been used to increase this research at NAU and combine this with records of faculty employment and research outcomes (publications and grant proposals). While the Partnership's objective of building capacity at an institutional level is also important, an analysis of the Partnership's success at that scale requires different data and is left to a different review.

We examine outcomes for individual faculty members affected by particular strategies: explicit recruitment to the institution, cultivating researchers from other disciplines already in the institution, mentoring programs within and across institutions, and investments in research infrastructure. We also describe two other strategies relevant to developing research capacity: engaging institutional administrators and planning for leadership succession.

Northern Arizona University is a medium-sized public university historically focused on undergraduate teaching. It is a member of the American Association of State Colleges and Universities and in the Carnegie classification scheme is a Doctoral University - Higher Research Activity. It consistently ranks 200 or below in the list of institutions in total research and development funding (National Science Foundation 2017), with relatively few doctoral programs and graduates. In the collaboration discussed here, NAU teams with the University of Arizona's Cancer Center (hereafter UACC) to address specific aims in research, training, and community outreach related to cancer health disparities in minority communities in Arizona.

NCI Partnerships to Advance Cancer Health Equity (PACHE) were established in 2001 under the original title of Minority Institution - Cancer Center Partnerships. The 
program links minority-serving universities with cancer centers in developing capacity to address cancer health disparities, that is, disproportional impacts of cancer on under-served populations. Research projects are structured as collaborations, typically with less experienced researchers at the minority-serving institution paired with established researchers at the cancer center. Faculty teams are supported for pilot projects for up to 3 years and are expected to build preliminary results and publications leading to greater funding for up to 3 additional years. Funds are also directed by the partnerships to recruiting and training diverse students and early-career researchers in cancer-related work. Finally, since the focus is on cancer disparities, each partnership does outreach with affected communities through community-based, participatory research and community health educators, ensuring engagement with and reporting to the communities of focus.

Our Partnership (NACP) was initially funded in 2002; in 2007 the initial five years of funding ended. After two years of interim activities, a successful competing renewal proposal was funded in 2009 for another five years. A third successful proposal was funded in 2014, and NACP is consequently in its third five-year funding period. Over the past six years, an extensive evaluation effort has provided detailed information for formative and summative purposes (Trotter et al. 2015), including typical outcomes such as publications, grant proposals, and student mentoring. For our purposes in this article, our outcomes were defined as follows. For all NAU faculty members supported by the Partnership, either as a principal investigator or as a research project leader, we addressed the following questions:

- Did the faculty member go through any promotion or tenure applications during or after the period of NACP support, and if so, was the application successful? Successful promotion would indicate a positive impact on the university's and individual's research capacity.

- Is the faculty member still employed at NAU in fall 2015? Long-term retention of the faculty member at the university would indicate a positive impact on university capacity. On the other hand, departure from the university would indicate that the NACP investment did not result in a long-lasting increase in university capacity.

- Has the researcher authored scholarly publications during or after the period of support? Increasing faculty publication in the peer-reviewed literature would be a positive outcome.

- Has the faculty member submitted external grant proposals, other than for direct NACP pilot or full projects, through NAU? Have any of those grant proposals been funded? Obtaining external grant funding is a common indicator of an individual's capacity as a researcher.

The first two questions were addressed by examining NACP files and soliciting CV's from previously-supported faculty members, in some cases reaching those who had departed NAU through internet searching. The third question was addressed by consulting two on-line bibliographic databases, PubMed Central and Web of Science тм from Thomson-Reuters. The fourth question was addressed through a search of institutional data held in the NAU Office of Sponsored Projects. We consulted with the NAU Institutional Review Board before seeking institutional records; the IRB confirmed that this case study is not a human subjects research project. We compiled and examined outcomes at the level of individual faculty members, but in accord with 
IRB guidance we present results only in aggregated summaries so that the identity of any individual faculty member cannot be not revealed.

\section{Overall Outcomes for NACP-Supported Faculty Members}

During the first two cycles of funding at NAU, the Partnership supported twenty-one (21) individuals either as research project leaders, principal investigators, or program leaders for development, training, or outreach. Outcomes for individuals vary, and the success of the project in achieving certain outcomes has been less than uniformly high. Overall, the Partnership has had more positive impact on some outcomes than on others.

\section{Promotion and Tenure Applications}

NACP participants have generally enjoyed positive outcomes for promotion and tenure decisions. Individuals hired from outside NAU in the NACP and other pre-tenure individuals who received NACP support have all been successful in assistant-toassociate professor promotion and tenure. Several individuals supported as assistant or associate professors also moved successfully through promotion to full professor. One non-tenure-track research assistant professor was successful in promotion to research associate professor. Several individuals successfully applied for and moved into leadership or administrative positions at the departmental or central university level. In all, 11 of the 21 participants had positive outcomes in these career steps, and no participating individuals were denied promotion or tenure. In sum, NACP experience has been positively correlated with career advancement within NAU.

\section{Continued Employment at the University}

Of the 21 individuals supported over the 13 years reviewed, four are still employed at NAU and actively supported on NACP funding, either as research project leaders or as NACP principal investigators and program leaders. Of the 17 persons whose NACP support has ended, one departed from NAU within one year after the last date of NACP support; seven had departed from NAU within 5 years of the end of support, and only 5 have been retained as long as 10 years after the end of NACP participation. Some departures were retirements, but at least four were moves of early or midcareer individuals to research positions elsewhere. While individuals were successful in developing research and advancing their careers, these departures represent a disappointing outcome in terms of long-lasting increases in the university's research capacity.

\section{Peer-Reviewed Publications}

Because of the disciplinary breadth of faculty participants, we searched for publications both in the PubMed Central ${ }^{\circledR}$ database (www.ncbi.nlm.nih.gov/pmc/) and in the Thomson-Reuters Web of Science тм bibliographic database. Five of the 21 participants were not represented by any publications dated after initial support from NACP. Three individuals had publications in one or both databases on topics not related to 
the aims of NACP (that is, not related to cancer, to health disparities, to community outreach and community-based participatory research, or to biomedical training activities). While the remaining 13 individuals had authored publications in one or both databases for the period under review, many of the papers did not pertain to NACP supported work; only 6 individuals cited NACP support for relevant work. In conclusion, there is little evidence in the record that NACP funding directly facilitated publication productivity.

\section{External Grant Proposals and Funding}

We examined data on grants received through the NAU Office of Sponsored Projects, by investigator, for the time period since each individual's initial support by NAU. Of the 21 supported individuals, 9 received no external funding during or after NACP support. Ten did have external funding for projects related to biomedical or health disparities, and two others received funding for projects not in biomedical or health disparities fields. While established faculty members may have had successful funding records prior to association with NACP, some had not previously been funded by NIH or other biomedical entities. Early career individuals supported as research project leaders were generally successful in obtaining external funding after NACP support: 9 of 10 funded externally, 7 from biomedical agencies. It appears that Partnership support did lead to increased levels of external funding for biomedical and health disparities work for some but not all participants.

\section{Strategies Attempted, Results, and Challenges}

\section{Recruiting New Faculty from outside the Institution}

The Partnership has twice allocated funding to recruitment of new tenure-track faculty, including funds for salary and benefits prior to NAU picking up the lines. In the early years two faculty members were recruited with explicit expectations of pursuing cancer-related research; the grant provided recruiting expenses, startup, and an initial two years of salary. The faculty members received release time from teaching while supported on the grant (a novel practice for NAU at that time) and were assisted in developing collaborations for pilot projects. Both found collaborators at UACC and submitted pilot and full grant proposals. Both were successful in recruiting minority students into their labs at the undergraduate and/or masters' level. One was successful in publishing peer-reviewed papers on Partnership work; however, neither investigator garnered external funding for cancer-related research. One faculty member left NAU for another academic institution; the other remains but is no longer conducting cancer research.

NAU also provided institutional funds to support hires in areas of cancer or health equity research without using Partnership funds. In one case a position was provided to recruit a minority faculty member who was subsequently supported by NACP before generating external support for independent research. This faculty member maintains a productive research program but no longer collaborates directly with UACC investigators on the original project. In a second case a department voluntarily recruited a research active faculty member with an interest in cancer health equity. This faculty member has been unsuccessful in 
finding a co-investigator at $\mathrm{UACC}$, but is developing research projects that include minority health equity. In a third case, Partnership support was used to increase the startup package to recruit a mid-career faculty member with prior cancer-related research experience. This person has now earned support as co-leader of an NACP pilot project. Institutional investments, then, have proven to have more positive sustained impact on cancer research capacity than grant-funded recruitments.

In today's constrained financial conditions, state and minority institutions frequently have limited ability to create new faculty lines or to redirect lines from teaching to research. Even talented researchers may find it difficult to achieve productivity in a new context, especially if institutional resources are limited or if departmental and institutional cultures have not yet evolved to support research. Finally, productive researchers can be recruited away to more research-active settings or to institutions with greater financial resources, making successful retention as important as hiring. We conclude that the conventional approach to building research through new hiring is neither easy nor consistently successful.

\section{Cultivating and Attracting Researchers from within Existing Faculty}

As noted, it may not be feasible for many institutions to rely solely upon creating large numbers of new faculty positions in order to build research activity. Drawing upon existing faculty strengths and encouraging already active researchers to take on new research activities is an unconventional alternative that the Partnership has pursued by using several mechanisms.

Call for Proposals for Pilot Projects The NACP budget included funds for new pilot project collaborations in 6 of the 13 years covered in this review. We disseminated a request for proposals at both universities. Guidelines require a collaboration involving at least one member from each institution; one challenge is that many faculty members at one institution are not acquainted with potential collaborators at the other. Establishing a letter-of-intent stage, where individuals indicate the general nature of their interests, has allowed us to carry out match-making activities in time for potential collaborators to meet one another and carry out initial planning prior to submitting a pilot proposal. This step has increased the number of faculty members at each institution expressing interest in NACP and the number of pilot project proposals submitted from 1 to 2 proposals to roughly a dozen per deadline.

Summer Research Conference Nine years after the Partnership began, NACP initiated an annual summer research conference. The initial aims were to provide an opportunity for all NACP-supported researchers to present ongoing work for feedback and to allow potential collaborators from NAU and UACC to become familiar with NACP. Thanks to feedback from an engaged evaluation process, the conference has sometimes also provided opportunities for active work on manuscripts, for exposure to minority cultural perspectives and research priorities, and for personnel across the large project to interact. Two funded projects involving summer conference attendees have now emerged, so this strategy has demonstrated some success.

Planning Grants For proposals deemed promising but premature, modest funding is provided on occasion for pairs of researchers to meet for collaborative discussions and 
to carry out proof of concept studies. These planning grants strengthen proposals from the team in subsequent rounds to meet the expectation that applications submitted internally can be reviewed as rigorously as an externally-submitted NIH proposal. This mechanism has been highly effective; in years 12 and 13 the investigators leading all funded pilot and full projects had earlier received planning grant funds to carry out pilot studies, develop Institutional Review Board protocols, and carry out other work to strengthen the eventual proposal.

Affiliates Program In 2013 the Partnership initiated a formal Affiliates Program at NAU (later adopted by the UACC). We identify and provide modest support for faculty members who have cancer research backgrounds or whose history of student mentoring is a good fit for NACP. Affiliates are encouraged to participate in NACP or other cancer-related meetings and activities. They are supported to travel to the other institution to present seminars and seek collaborators. They are also invited to submit proposals when pilot funds are available, and several have successfully moved to funded status. This low-cost program has increased the number of faculty members at NAU actively cultivating collaborations and cancer-related funding opportunities.

Advisory Committee Member Engagement One novel phenomenon has generated additional research projects: the transition of Internal Advisory Committee (IAC) members to become project co-leaders. NCI requires PACHE partnerships to have an IAC, comprising individuals from both institutions who review progress annually and assist with planning. NAU selects IAC members from the ranks of institutional administrators such as associate vice presidents for research, department chairpersons or deans. In several cases IAC members have become enthusiastic about potential research and/or potential collaborators they have met through NACP. As they have formulated plans to apply for potential NACP funding, they have resigned IAC membership in order to avoid conflicts of interest. One lead investigator, two program leaders, and three research project co-leaders have come from our IAC.

Mentoring Programs within and across Institutions The standard mentoring approach within the UACC entails pairing new faculty members with experienced faculty researchers in a mentoring committee advising on career and research. The committee meets twice a year with the mentee to discuss progress and set goals, to provide guidance on lab management and mentoring trainees, and to familiarize the mentee with criteria for promotion and tenure. The UACC has also developed a mechanism for increasing the competitiveness of grant proposals by providing a review prior to submission. In early years the Partnership used this model to link senior UACC mentors with new NAU researchers.

Conventional mentoring, however, was not sufficient to overcome two significant challenges in this Partnership: geographic distance and difference in institutional cultures. Northern Arizona University in Flagstaff is more than 250 miles from the UACC in Tucson. Initially mentoring pairs met only infrequently, usually in NACP meetings where other objectives reduced time available for personal consultation. Differences in context between the two universities pose even greater challenges to effective mentoring. Historical emphasis at NAU on instruction and work with undergraduates can cause conflict between growing research expectations for NACP- 
supported faculty members and the expectations of their home departments. Mentors from the UACC were initially unfamiliar with promotion and tenure criteria at NAU and could not always give relevant advice. Other issues at NAU not understood by UACC mentors are lack of infrastructure, fewer research-active colleagues for support, fewer doctoral students, and minimal access to modern equipment. Meanwhile, most UACC researchers must provide a significant portion of their own salaries through grants and therefore spend much time writing proposals. UACC faculty members can view the effort needed to develop functional partnerships as time taken from higher priorities.

We now routinely use video-conferencing to overcome the distance barrier. Video meetings also enable junior faculty from NAU to participate in the Young Investigator's Forum at the UACC, linking those early career individuals with senior researchers at UACC for advice on new research proposals. We emphasize positive elements of the cross-institutional structure, including specific experimental and analytical capabilities at NAU that are useful to UACC researchers. Importantly, NAU faculty members often have more extensive experience and understanding of issues related to minority students. On occasion, pre-tenure individuals at UACC have collaborated with more established researchers at NAU. Overall the Partnership promotes the idea that mentoring is not a simple uni-directional or top-down relationship.

The Partnership evolved over time to provide more guidance for faculty development by assigning a senior researcher from each institution as a "research development" principal investigator. These individuals carry out several activities aimed at supporting the professional development of researchers while also cultivating new collaborations and the general increase of cancer-related research capacity at NAU (Table 1). The Partnership also brings department chairs and deans into mentoring conversations at NAU in order to achieve protected time and resources for faculty members to conduct research and to write grants and manuscripts.

\section{Investing in Research Infrastructure}

Investment in research support has changed as funded research projects have evolved. Early projects were laboratory-based, focusing on environmental health effects and providing an opportunity to invest in research infrastructure such as equipment that would support all NACP work. Funds were also provided for a research technician to support the projects. As NACP-funded research transitioned to include community-based and health sciences work, research needs could no longer

Table 1 Responsibilities of principal investigators responsible for expanding research capacity

Assist faculty members in finding potential collaborators at the sister institution.

Assist researchers to refine research projects to meet goals of Partnership.

Coordinate reviews of new and continuing pilot projects (by ad hoc reviewers and by advisory committee members).

Maintain attention to the expectations for research progress.

Collaborate with the Outreach Core to receive input from minority communities.

Collaborate with the Training Core to guide faculty development and faculty work with research students. 
be met by such focused investments; and we are currently exploring ways of providing staff support for behavioral and population-focused research.

Another important innovation at NAU has been a focus on access to research students. Several NAU departments with faculty members supported by the Partnership do not have Ph.D. or even M.S. programs, and faculty members in these units have typically had only undergraduates or no research students at all in their labs. We have linked several faculty members to M.S. and Ph.D. students from other programs, with subsequent acceleration of data collection and publication.

\section{Investing in Institutional Leadership}

An important element of the Partnership's long-term success has been building strong sustained relationships with NAU's administrative leaders. From the beginning, deans and department chairs have been included as members of the Internal Advisory Committee. Their participation provides helpful insights about factors shaping the development of research capacity in their units and in the institution; as noted above, it is beneficial for early-career researchers to have their academic supervisors engaged with Partnership expectations and opportunities.

We have also benefited from higher-level connections with central administrators and the state Board of Regents. One of us (Huenneke) has been lead investigator for the Partnership while simultaneously serving as NAU's vice president for research and then provost. These connections have created opportunities to seek university resources and to align NACP's activities realistically with priorities of the University's governing board. On the other hand, having an institutional leader as program leader does create tensions in terms of workload and potential perceived conflicts of interest. Including central administrators on the IAC is another tactic for strengthening alignments. Finally, we report annually on NACP activities and accomplishments to the presidents, provosts, and vice presidents for research of both parent institutions; these reports underlie a systematic series of conversations with institutional leaders in order to ensure support and to overcome institutional barriers to continued research capacity development.

\section{Planning for Leadership Succession and Transition}

Like any long-lived project, the Partnership has experienced transitions in leadership for core activities over its history. While funding agencies require a rationale for assembling the leadership team for any major proposal, in our experience there is often little attention given to leadership development or planned transitions. Yet any project dedicated to faculty career development ought to look ahead to possible transitions and departures. Over 13 years the Partnership has seen several investigators leave NAU for career opportunities at other institutions, and leaders have changed as interests evolved. We have now developed formal planning for potential personnel changes. For example, advancement of the Training Core PI into the role of Lead Investigator was foreseen and prepared for by formal participation in University leadership and supervisor training. NACP principal investigators regularly assess research project leaders and Internal Advisory Committee members as potential senior leaders. Incorporating explicit discussion of chances for advancement and 
leadership opportunities into our mentoring sessions with researchers has proven important in retaining talented junior faculty. Our evaluation process collects data on communication and relationship interactions of all Partnership members; this social network analysis provides insights into individuals who might reasonably be able to step into a new leadership role (Trotter et al. 2015).

\section{Discussion}

\section{Lessons Learned}

Our experiences in sustaining this long-term effort to build research capacity in a public university have led us to some conclusions about helpful approaches. We believe that the suggestions below merit further thought and study.

1) Institutional investment is as (or more) important than external funding for recruiting new faculty members. Long-term faculty success within the university setting relies upon the kind of "buy-in" obtained only when the university (or a component unit) has formally invested its resources in individuals.

2) Programs should attend to career development plans for all, not just for junior faculty, and adapt plans for the particular institution and program. Moreover, institutions should explore and cultivate talent within established faculty members as well as new recruits; they represent reservoirs of talent and energy, and frequently modest assistance can enable them to step up research productivity and take on program leadership.

3) The conventional mentoring model is not always viable and can be too constraining. Our experience suggests that the minority-serving institution may have expertise and unique facilities that are beneficial to researchers at the larger, more research-focused university. Typically the minority-serving institution also has expertise and appropriate cultural and organizational approaches for working effectively with under-represented minority students. The Partnership's goals of expanding cancer-related research and training have been most effectively achieved by permitting and supporting more diverse team structures.

4) Establishing initial success for a researcher is necessary but not sufficient. We have learned that it is crucial to attend to researchers' longer term connections to and opportunities within the institution. In particular, research success may well increase the chance that a faculty member might be recruited by another institution; and initial investments may not be sufficient to retain a researcher long-term without attention to engagement and career development. Furthermore, projects such as NACP cannot fund faculty members directly for long periods; after a project ends, researchers become vulnerable to being lost from the institution. It is therefore imperative to develop strategies that can keep individuals engaged and successful over the longer term.

5) Deliberate attention to inviting advisory committee members and institutional leaders can pay off greatly. Including key deans and research office personnel on our internal advisory committee has been effective in assuring support (or at least understanding) of participating research faculty members. Strategic thought should go into the support, training, and engagement of advisory committee members to ensure the connections are deep and genuine. Our advisory committee members have in some cases even moved into direct leadership roles in the project. 
6) Frank acknowledgment of differing institutional cultures and values is essential; and programs must be flexible in choosing relevant, important metrics for program evaluation and management. Even if a single set of overall program goals governs expectations for research teams, "one size fits all" approaches to supporting productive outcomes will not be successful in the face of differing departmental and college histories and contexts.

7) Transition and succession planning should be considered from the start. Any long-term program will experience changes in leadership and in senior personnel; successful individuals in particular can be recruited to other institutions. Individuals may also experience the desire to shift roles or to devote more energy to other priorities. Management teams and advisory groups should continuously assess and cultivate potential leaders.

\section{Questions for Formal Study}

This review has stimulated us to consider a more formal effort to evaluate some interesting hypotheses, and we invite others to explore the questions we pose below.

- How do gender and ethnic/racial status influence the faculty experience within the PACHE program? We hypothesize that individuals from under-represented groups experience higher internal satisfaction and perhaps greater institutional support for participation in such partnerships than do majority individuals.

- What is the impact of institutional context (cancer center vs. minority serving institution, medical school or no medical school) on a faculty member's experience within the Partnership? We expect different faculty reward systems in major research institutions vs. undergraduate-focused departments in minority-serving institutions. We hypothesize that individuals in minority-serving institutions might experience more dissonance and less institutional reward or support for research success within the Partnership.

- Do training or community outreach components of these partnerships offer different levels of personal or professional satisfaction for faculty members in the cancer centers vs. those in the MSI? Do perceptions differ among faculty individuals by gender or ethnic/racial identity?

- What is the role of the partnership in generating increased research capacity beyond cancer, especially in the minority-serving institution? We seek to understand the contexts within which a specific funded program such as PACHE might result in broader gains in numbers and productivity of faculty researchers, both within and beyond departments with direct support.

\section{Conclusion}

As noted in our introduction, many universities face pressure to expand their research activities. Research universities have many options for crafting specific policies and strategies for increasing research productivity (Cole 2007; Fisher 2009; Hearn et al. 2006), especially when there is interest in a specific discipline or type of work such as the community-based health equity research prioritized by the PACHE programs (see Harkavy and Hartley 2012, for a similar example). We encourage systematic, research-based reviews of the success and 
impact of these targeted university and federal investments as a desirable way of helping institutional leaders make the most of existing and future programs.

Acknowledgments This work grew out of NAU's experience with The Partnership for Native American Cancer Prevention (in an earlier phase, the Native American Cancer Research Partnership). The Partnership has been funded by grant numbers UU54CA 96281 and U54CA 143924 (for the University of Arizona Cancer Center), and U54CA 96320 and U54CA 143925 (to NAU), under the Comprehensive Partnerships to Advance Cancer Health Equity of the National Institutes of Health. This content is solely the responsibility of the authors and does not necessarily represent the official views of the National Institutes of Health. We are grateful to current and former participants in NACP programs for sharing their CV's and their personal experiences with us. Finally, we thank members of the Internal Advisory Committee and the (external) Program Steering Committee for their long-term support of and assistance to the Partnership and its many faculty, staff, and student members.

Open Access This article is distributed under the terms of the Creative Commons Attribution 4.0 International License (http://creativecommons.org/licenses/by/4.0/), which permits unrestricted use, distribution, and reproduction in any medium, provided you give appropriate credit to the original author(s) and the source, provide a link to the Creative Commons license, and indicate if changes were made.

\section{References}

Arizona Board of Regents (2015). Annual report, FY 2015. Retrieved from https://www.azregents.edu/sites/ default/files/public/Impacting\%20Arizona\%20-\%20Arizona\%20Board\%20of\%20Regents\%20Annual\%20 Report\%20for\%20FY\%202015.pdf

Birx, D. L., Anderson-Fletcher, E., \& Whitney, E. (2013). Growing an emerging research university. Journal of Research Administration, 44(1), 11-35.

Bosch, A., \& Taylor, J. (2011). A proposed framework of institutional research development plans. Journal of Higher Education Policy and Management, 33, 443-457.

Christensen, C. M., \& Eyring, H. J. (2011). The innovative university: Changing the DNA of higher education from the inside out. San Francisco, CA: Jossey-Bass.

Cole, S. S. (2007). Research administration as a living system. Journal of Research Administration, $38(2), 14-27$

Fisher, R. (2009). A framework for research at Canadian colleges. The College Quarterly, 12(4), 1-32. Retrieved from http://files.eric.ed.gov/fulltext/EJ889560.pdf

Fowles, J. (2014). Funding and focus: Resource dependence in public higher education. Research in Higher Education, 55, 272-287.

Godreau, I., Gavillan-Suarez, J., Franco-Ortiz, M., Calderon-Squiabro, J. M., Marti, V., \& Gaspar-Concepcion, J. (2015). Growing faculty research for students' success: Best practices of a research institute at a minorityserving undergraduate institution. Journal of Research Administration, 46(2), 55-78.

Harkavy, I., \& Hartley, M. (2012). Integrating a commitment to the public good into the institutional fabric: Further lessons from the field. Journal of Higher Education Outreach and Engagement, 16(4), 17-36.

Hearn, J. C., Lewis, D. R., Kallsen, L., Holdsworth, J. M., \& Jones, L. M. (2006). "Incentives for managed growth": A case study of incentives-based planning and budgeting in a large public research university. Journal of Higher Education, 77, 286-316.

Jaquette, O., \& Curs, B. R. (2015). Creating the out-of-state university: Do public universities increase nonresident freshman enrollment in response to declining state appropriations? Research in Higher Education, $56,535-565$.

Kornreich, L. D. (1973). University consortia: A unique approach to environmental education and research. Journal of the Air Pollution Control Association, 23, 755-760.

Kyvik, S., \& Aksnes, D. W. (2015). Explaining the increase in publication productivity among academic staff: A generational perspective. Studies in Higher Education, 40, 1438-1453.

Litwin, J. M. (2009). The efficacy of strategy in the competition for research funding in higher education. Tertiary Education and Management, 15, 63-77.

National Cancer Institute (2015). Comprehensive Partnerships to Advance Cancer Health Equity (CPACHE, U54). PAR-15-103. Retrieved from http://grants.nih.gov/grants/guide/pa-files/PAR-15-103.html

National Center for Educational Statistics (2015). Digest of educational statistics, 2014. Table 333.30, Appropriations from state and local governments for public degree-granting postsecondary institutions, by 
state or jurisdiction: Selected years, 1990-91 through 2012-13. Retrieved from https://nces.ed. gov/programs/digest/2014menu_tables.asp

National Science Foundation (2017). National Center for Science and Engineering Statistics, Academic institution profiles, Rankings by total $R \& D$ expenditures. Retrieved from https://ncsesdata.nsf.gov/profiles/site?method= rankingBySource \&ds=herd

Neumann, A., \& Terosky, A. L. (2007). To give and to receive: Recently tenured professors' experiences of service in major research universities. Journal of Higher Education, 78, 282-310.

Romainville, M. (1996). Teaching and research at university: A difficult pairing. Higher Education Management, $8,135-144$.

Rosenzweig, R. M. (1987). Universities move toward new responsibilities in a more complex environment. Research Management Review, 1, 61-67.

Selingo, J. J. (2013). College (un)bound: The future of higher education and what it means for students. New York, NY: Houghton Mifflin Harcourt.

Shehzad, U., Fareed, Z., Zulfiqar, B., Shahzad, F., \& Latif, H. S. (2014). The impact of intellectual capital on the performance of universities. European Journal of Contemporary Education, 10, 273-280.

Slaughter, S., \& Rhoades, G. (2004). Academic capitalism and the new economy: Markets, states, and higher education. Baltimore, MD: The Johns Hopkins University Press.

Streharsky, C. J. (1991). Evolution of the university research mission in the United States. Research Management Review, 5, 35-50.

Taylor, B. J., \& Cantwell, B. (2015). Global competition, U.S. research universities, and international doctoral education: Growth and consolidation of an organizational field. Research in Higher Education, 56, 411-441.

Thompson, B., O’Connell, M., Loest, H., Anderson, J., \& Westcott, R. (2013). Understanding and reducing obstacles in a collaboration between a minority institution and a cancer center. Journal of Health Care for the Poor and Underserved, 24, 1648-1656.

Trotter II, R. T., Laurila, K., Alberts, D., \& Huenneke, L. F. (2015). A diagnostic evaluation model for complex research partnerships with community engagement: The partnership for native American cancer prevention (NACP) model. Evaluation and Program Planning, 48, 10-20.

Warshaw, J. B., \& Hearn, J. C. (2014). Leveraging university research to serve economic development: An analysis of policy dynamics in and across three U.S. states. Journal of Higher Education Policy and Management, 36, 196-211. 